\title{
Collective Hamiltonian for chiral modes
}

\author{
Q. B. Chen, ${ }^{1}$ S. Q. Zhang, ${ }^{1, *}$ P. W. Zhao, ${ }^{1}$ R. V. Jolos,${ }^{2,3}$ and J. Meng ${ }^{1,4,5,+}$ \\ ${ }^{1}$ State Key Laboratory of Nuclear Physics and Technology, \\ School of Physics, Peking University, Beijing 100871, China \\ ${ }^{2}$ Joint Institute for Nuclear Research, Dubna 141980, Russia \\ ${ }^{3}$ Institut für Kernphysik der Universität zu Köln, Köln 50937, Germany \\ ${ }^{4}$ School of Physics and Nuclear Energy Engineering, \\ Beihang University, Beijing 100191, China \\ ${ }^{5}$ Department of Physics, University of Stellenbosch, Stellenbosch, South Africa
}

(Dated: July 24, 2018)

\begin{abstract}
A collective model is proposed to describe the chiral rotation and vibration and applied to a system with one $h_{11 / 2}$ proton particle and one $h_{11 / 2}$ neutron hole coupled to a triaxial rigid rotor. The collective Hamiltonian is constructed from the potential energy and mass parameter obtained in the tilted axis cranking approach. By diagonalizing the collective Hamiltonian with a box boundary condition, it is found that for the chiral rotation, the partner states become more degenerate with the increase of the cranking frequency, and for the chiral vibrations, their important roles for the collective excitation are revealed at the beginning of the chiral rotation region.
\end{abstract}

PACS numbers: 21.60.Ev, 21.10.Re, 23.20.Lv

*Electronic address: sqzhang@pku.edu.cn

$\dagger$ Electronic address: mengj@pku.edu.cn 


\section{INTRODUCTION}

Since the chirality in nuclear physics was originally suggested by Frauendorf and Meng in 1997 [1], it has been one of the hot topics in nuclear physics. The corresponding experimental signals, the chiral doublet bands, were first observed in $N=75$ isotones in 2001 [2], and so far have been extensively reported in more than 30 nuclei.

For a rotating nucleus with triaxiality, the three angular momenta, including collective rotor angular momentum along the intermediate axis, together with angular momenta of the valence particles (holes) along the nuclear short (long) axis, can construct the chiral geometry [1, 2]. It has been demonstrated that the chiral doublet bands generally start from a left-right chiral vibration mode and evolve into a static chiral mode with the increase of $\operatorname{spin}[3,4]$.

Theoretically, chiral doublet bands were first predicted by the tilted axis cranking (TAC) approach and particle rotor model (PRM) [1]. Subsequently, numerous efforts have been devoted to the development of both the TAC methods [5 7] and PRM models [4, 8, 9]. Both PRM and TAC have their own advantages and disadvantages. The PRM is a quantal model consisting of the collective rotation and the intrinsic single-particle motions, and the total angular momentum is a good quantum number. It describes a system in the laboratory reference frame where the spontaneously broken chiral symmetry in the intrinsic reference frame is restored. In the PRM, the energy splitting and quantum tunneling between the doublet bands can be obtained directly. However, rigid rotor with quadrupole deformation parameters $\beta$ and $\gamma$ has to be assumed at the very beginning in the practical PRM calculation.

TAC approach is based on mean-field theory, and permits the calculation for the orientation of the density distribution relative to the angular momentum vector. However the TAC cannot describe the energy splitting and the quantum tunneling between the chiral doublet bands [1]. Up to now, the TAC method based on the Woods-Saxon or Nilsson potential [5], as well as the microscopic self-consistent Skyrme Hartree-Fock model [6, 7] have been devoted to study the chirality.

To describe the energy splitting between the chiral doublet bands, one has to go beyond the mean-field approximation. So far, this has been done in the framework of tilted axis cranking plus the random phase approach (RPA) model [3, 10]. However, this model is 
restricted in the description of the chiral vibration since the quantum tunneling in the chiral rotation is beyond the realm of RPA. Therefore, it is imperative to search a unified method for studying both chiral rotation and vibration in the framework of TAC.

In analogy to Bohr Hamiltonian, constructing a collective Hamiltonian on the TAC solutions provides one of the ways out. Instead of the $\beta$ and $\gamma$ degrees of freedom in Bohr Hamiltonian, a chiral degree of freedom should be introduced. Subsequently, the quantal tunneling in the region of chiral rotation can be described by considering the chiral fluctuations around mean-field minima besides the region of chiral vibration naturally described by the collective Hamiltonian.

In this work the collective Hamiltonian for a system of one $h_{11 / 2}$ proton particle and one $h_{11 / 2}$ neutron hole coupled to a triaxial rigid rotor is constructed. The potential energy and mass parameters involved in the collective Hamiltonian are extracted from the TAC calculations. By diagonalizing the collective Hamiltonian, the energy levels and wave functions are calculated and discussed in detail. The paper is organized as follows. In Sec. II, based on the tilted axis cranking approach, the procedures for constructing and solving the collective Hamiltonian are introduced. The numerical details are presented in Sec. III. In Sec. IV] the potential energy and mass parameters obtained from TAC approach are shown and the corresponding energy levels and wave functions obtained from the collective Hamiltonian are discussed in details. Finally, the summary is given in Sec. V.

\section{THEORETICAL FRAMEWORK}

\section{A. Tilted axis cranking model}

The detailed formalism for TAC can be found in Ref. [11]. For a schematic discussion, similar as Ref. [1], we consider a system of the $h_{11 / 2}$ proton particle and the $h_{11 / 2}$ neutron hole coupled to a triaxial rigid rotor. The cranking Hamiltonian reads

$$
\begin{aligned}
\hat{h}^{\prime} & =\hat{h}_{\text {def }}-\vec{\omega} \cdot \hat{\vec{j}}, \\
\hat{\vec{j}} & =\hat{\vec{j}}_{\pi}+\hat{\vec{j}}_{\nu}, \\
\vec{\omega} & =(\omega \sin \theta \cos \varphi, \omega \sin \theta \sin \varphi, \omega \cos \theta),
\end{aligned}
$$


where the Hamiltonian of the deformed field is $\hat{h}_{\mathrm{def}}=\hat{h}_{\mathrm{def}}^{\pi}+\hat{h}_{\mathrm{def}}^{\nu}$ with the single- $j$ shell Hamiltonian

$$
\hat{h}_{\mathrm{def}}^{\pi(\nu)}= \pm \frac{1}{2} C\left\{\left(\hat{j}_{3}^{2}-\frac{j(j+1)}{3}\right) \cos \gamma+\frac{1}{2 \sqrt{3}}\left(\hat{j}_{+}^{2}+\hat{j}_{-}^{2}\right) \sin \gamma\right\} .
$$

The TAC solutions are obtained self-consistently by minimizing the total Routhian surface

$$
E^{\prime}(\theta, \varphi)=\left\langle h^{\prime}\right\rangle-\frac{1}{2} \sum_{k=1}^{3} \mathcal{J}_{k} \omega_{k}^{2},
$$

with respect to the angles $\theta$ and $\varphi$, where the moments of inertia for irrotational flow are adopted, i.e.,

$$
\mathcal{J}_{k}=\mathcal{J}_{0} \sin ^{2}\left(\gamma-\frac{2 \pi}{3} k\right)
$$

As discussed in Ref. [1], there are three kinds of different solutions which can be distinguished by the different values of $\theta$ and $\varphi$ : a) Principal axis cranking (PAC) solution: $\theta=0, \pi / 2, \varphi=0, \pm \pi / 2$. b) Planar TAC solution: $\theta \neq 0, \pm \pi / 2, \varphi=0, \pm \pi / 2$ or $\theta=\pi / 2$, $\varphi \neq 0, \pm \pi / 2$. c) Aplanar TAC solution: $\theta \neq 0, \pi / 2, \varphi \neq 0, \pm \pi / 2$, i.e., the chiral solution.

In Ref. [12], an orientation operator $\hat{\sigma}$ was introduced to characterize the chiral degree of freedom. In the TAC approach for chiral solutions, the orientation operator is written as

$$
\hat{\sigma}=\left(\hat{\vec{j}}_{\pi} \times \hat{\vec{j}}_{\nu}\right) \cdot \hat{\vec{R}}=\left|\hat{\vec{j}}_{\pi}\right| \cdot\left|\hat{\vec{j}}_{\nu}\right| \cdot|\hat{\vec{R}}| \cdot \sin \theta_{\mathrm{PN}} \cdot \sin \theta \sin \varphi
$$

in which $\theta_{\mathrm{PN}}$ denotes the angle between of $\hat{\vec{j}}_{\pi}$ and $\hat{\vec{j}}_{\nu}$. This operator has opposite signs for the left-handed and the right-handed systems. Follow Ref. [1], we restrict the angle $\theta$ to $0 \leq \theta \leq \pi / 2$, and by varying $\varphi$ from $-\pi / 2$ to $\pi / 2$, we get $\varphi$ as a dynamical variable describing a transition from the left-handed to the right-handed system. Thus for a given $\theta$, the angle $\varphi$ can be used to characterize the chiral degree of freedom. We note that the calculations performed in Refs. [5] and [10] for the potential energy as a function of $\theta$ and $\varphi$ for ${ }^{134} \operatorname{Pr}$ have shown a softer nature of the potential in the $\varphi$ direction compare to the $\theta$ direction.

\section{B. Collective Hamiltonian}

Based on the TAC model, a collective Hamiltonian including the chiral degree of freedom could be constructed. The classical form of a collective Hamiltonian can be written in terms of $\varphi$ as

$$
H_{\mathrm{coll}}=T_{\mathrm{vib}}(\varphi)+V(\varphi)
$$


where $\varphi$ is the variable which characterizes the chirality, and the potential energy $V(\varphi)$ could be extracted by minimizing the total Routhian Eq. (5) with respect to $\theta$ for given $\varphi$.

The vibrational kinetic energy of the chiral degree of freedom can be written as

$$
T_{\mathrm{vib}}=\frac{1}{2} B \dot{\varphi}^{2},
$$

where $B$ is the mass parameter. The Hamiltonian (8) is quantized according to the general Pauli prescription [13], i.e., for a classical kinetic energy,

$$
T=\frac{1}{2} \sum_{i j} B_{i j}(q) \dot{q}_{i} \dot{q}_{j}
$$

the corresponding quantized form reads

$$
\hat{H}_{\text {kin }}=-\frac{\hbar^{2}}{2} \frac{1}{\sqrt{\operatorname{det} B}} \sum_{i j} \frac{\partial}{\partial q_{i}} \sqrt{\operatorname{det} B}\left(B^{-1}\right)_{i j} \frac{\partial}{\partial q_{j}} .
$$

For the vibrational kinetic energy in Eq. (9), the mass parameter takes a $1 \times 1$ matrix form. Thus, the corresponding quantized form reads

$$
\hat{H}_{\text {kin }}=-\frac{\hbar^{2}}{2 \sqrt{B(\varphi)}} \frac{\partial}{\partial \varphi} \frac{1}{\sqrt{B(\varphi)}} \frac{\partial}{\partial \varphi} .
$$

Therefore, the quantized form of the collective Hamiltonian in Eq. (8) turns out to be

$$
\hat{H}_{\text {coll }}=-\frac{\hbar^{2}}{2 \sqrt{B(\varphi)}} \frac{\partial}{\partial \varphi} \frac{1}{\sqrt{B(\varphi)}} \frac{\partial}{\partial \varphi}+V(\varphi) .
$$

Note that the volume element in the present collective space is

$$
\int d \tau_{\text {coll }}=\int d \varphi \sqrt{B(\varphi)}
$$

and the quantized Hamiltonian of Eq. (13) is Hermitian with respect to the collective measure in Eq. (14).

\section{Mass parameter}

The details for calculating the mass parameter can be found in [14]. In this subsection, for completeness, a brief procedure is presented. Considering a variable $\varphi$ as a function of time, the time dependent Schrödinger equation is written as

$$
\hat{h}^{\prime}(t)|\psi(t)\rangle=i \hbar \frac{\partial}{\partial t}|\psi(t)\rangle .
$$


The general solution of this equation is

$$
|\psi(t)\rangle=\sum_{k} a_{k}(t) e^{i \phi_{k}(t)}|k\rangle
$$

where $\phi_{k}(t)=-\frac{1}{\hbar} \int_{0}^{t} E_{k}\left(t^{\prime}\right) \mathrm{d} t^{\prime}$ and $E_{k}(t)$ is the eigenvalue of the Hamiltonian $\hat{h}^{\prime}(t)$. By substituting Eq. (16) into Eq. (15), we obtain

$$
\dot{a}_{l}=-\dot{\varphi} \sum_{k} a_{k}(t) e^{i\left(\phi_{k}-\phi_{l}\right)}\left\langle l\left|\frac{\partial}{\partial \varphi}\right| k\right\rangle .
$$

We assume that the wave function is the lowest eigenstate of the cranking Hamiltonian $\hat{h}^{\prime}$ in Eq. (11) at $t=0$, i.e., $a_{k}=\delta_{k 0}$ at $t=0$. Here, $|0\rangle$ means the lowest state of $\hat{h}^{\prime}$. If we further assume that $a_{l \neq 0}$ are small and the variable $\varphi$ follows the relation of $\ddot{\varphi}=-\Omega^{2} \varphi$ with $\Omega$ being the vibrational frequency, the solution of the Eq. (17) can be obtained as

$$
a_{l}=\frac{i \hbar\left(E_{l}-E_{0}\right) \dot{\varphi}+\hbar^{2} \Omega^{2} \varphi}{\left(E_{l}-E_{0}\right)^{2}-\hbar^{2} \Omega^{2}}\left\langle l\left|\frac{\partial}{\partial \varphi}\right| 0\right\rangle e^{-i\left(\phi_{l}-\phi_{0}\right)},
$$

where $E_{l}$ and $E_{0}$ are the eigen energies of the cranking Hamiltonian $\hat{h}^{\prime}$ in Eq. (11).

The energy of the system can be written as

$$
\begin{aligned}
E(t) & =E_{0}+\sum_{l \neq 0}\left(E_{l}-E_{0}\right)\left|a_{l}\right|^{2} \\
& =E_{0}+\sum_{l \neq 0}\left(E_{l}-E_{0}\right) \frac{\left[\hbar^{2}\left(E_{l}-E_{0}\right)^{2} \dot{\varphi}^{2}+(\hbar \Omega)^{4} \varphi^{2}\right]\left|\left\langle l\left|\frac{\partial}{\partial \varphi}\right| 0\right\rangle\right|^{2}}{\left[\left(E_{l}-E_{0}\right)^{2}-\hbar^{2} \Omega^{2}\right]^{2}} \\
& =E_{0}+\frac{1}{2} B(\varphi) \dot{\varphi}^{2} .
\end{aligned}
$$

Neglecting the high-order terms $\left(\propto \varphi^{2}\right)$, the mass parameter can be obtained as

$$
\begin{aligned}
B(\varphi) & =2 \hbar^{2} \sum_{l \neq 0} \frac{\left(E_{l}-E_{0}\right)^{3}\left|\left\langle l\left|\frac{\partial}{\partial \varphi}\right| 0\right\rangle\right|^{2}}{\left[\left(E_{l}-E_{0}\right)^{2}-\hbar^{2} \Omega^{2}\right]^{2}} \\
& =2 \hbar^{2} \sum_{l \neq 0} \frac{\left(E_{l}-E_{0}\right)\left|\left\langle l\left|\left[\hat{h}^{\prime}, \frac{\partial}{\partial \varphi}\right]\right| 0\right\rangle\right|^{2}}{\left[\left(E_{l}-E_{0}\right)^{2}-\hbar^{2} \Omega^{2}\right]^{2}} .
\end{aligned}
$$

By substituting Eq. (11) into Eq. (20) and using the relation

$$
\left\langle l\left|\left[h_{\mathrm{def}}-\vec{\omega} \cdot \hat{\vec{j}}, \frac{\partial}{\partial \varphi}\right]\right| 0\right\rangle=\frac{\partial \vec{\omega}}{\partial \varphi}\langle l|\hat{\vec{j}}| 0\rangle,
$$

one obtains,

$$
B(\varphi)=2 \hbar^{2} \sum_{l \neq 0} \frac{\left(E_{l}-E_{0}\right)\left|\frac{\partial \vec{\omega}}{\partial \varphi}\langle l|\hat{\vec{j}}| 0\rangle\right|^{2}}{\left[\left(E_{l}-E_{0}\right)^{2}-\hbar^{2} \Omega^{2}\right]^{2}} .
$$


Once the vibrational frequency $\Omega$ is known, the mass parameter $B(\varphi)$ can be then determined by Eq. (22). Normally, $\Omega$ is determined as follows: a) for the chiral rotations, it is approximately taken as zero because the barrier penetration between the left-handed and right-handed states is low; b) for the chiral vibrations, the potential energy $V(\varphi)$ can be approximated by the harmonic oscillator potential $\frac{1}{2} K \varphi^{2}$ with the corresponding spring coefficient $K$. The mass parameter is

$$
B=\frac{K}{\Omega^{2}}
$$

Combining Eqs. (22) and (23), the value of $\Omega$ can be obtained for the chiral vibrations.

\section{Basis space}

After the mass parameter $B(\varphi)$ is obtained from the TAC model, the collective Hamiltonian (13) is constructed. It is easy to find that the collective Hamiltonian Eq. (13) keeps the parity conservation with respect to $\varphi \rightarrow-\varphi$. Therefore, the eigenstates of the collective Hamiltonian can be divided into two separate subspaces, i.e., the positive parity states and the negative parity states.

For the positive parity, the basis states can be taken as

$$
\psi_{n}(\varphi)=\sqrt{\frac{2}{\pi}} \frac{\cos (2 n-1) \varphi}{B^{1 / 4}(\varphi)}, \quad n \geq 1
$$

and for the negative parity they are

$$
\psi_{n}(\varphi)=\sqrt{\frac{2}{\pi}} \frac{\sin 2 n \varphi}{B^{1 / 4}(\varphi)}, \quad n \geq 1
$$

These basis states fulfill the box boundary condition,

$$
\psi_{n}(\pi / 2)=\psi_{n}(-\pi / 2)=0
$$

For the chosen basis, the normalization conditions with respect to the collective measure in Eq. (14) are satisfied.

The wave function can be then expanded by the basis states as

$$
\psi(\varphi)=\sum_{n=1}^{\infty} a_{n} \sqrt{\frac{2}{\pi}} \frac{\cos (2 n-1) \varphi}{B^{1 / 4}(\varphi)}+\sum_{n=1}^{\infty} b_{n} \sqrt{\frac{2}{\pi}} \frac{\sin 2 n \varphi}{B^{1 / 4}(\varphi)},
$$

where the expansion coefficients $a_{n}$ and $b_{n}(n \geq 1)$ are obtained by diagonalizing the collective Hamiltonian Eq. (13). 


\section{NUMERICAL DETAILS}

In the following calculations, the symmetric particle-hole configuration $\pi h_{11 / 2} \otimes \nu h_{11 / 2}^{-1}$ is considered and $\gamma$ deformation is assumed as $\gamma=-30^{\circ}$. The coupling parameters $C$ in the single- $j$ shell Hamiltonian are taken as $C_{\pi}=0.25 \mathrm{MeV}$ for the proton particle and $C_{\nu}=-0.25 \mathrm{MeV}$ for the neutron hole, respectively. The moment of inertia is chosen as $\mathcal{J}_{0}=40 \hbar^{2} / \mathrm{MeV}$. These parameters are the same as in Ref. [1].

\section{RESULTS AND DISCUSSION}

\section{A. Collective potential}

The potential energy surface in the rotating frame, i.e., the total Routhian $E^{\prime}(\theta, \varphi)$ as a function of $\theta$ and $\varphi$, is shown in Fig. 1] at the frequencies $\hbar \omega=0.1,0.2,0.3,0.4 \mathrm{MeV}$. The present results are consistent with those in Ref. [1] where the total Routhians surface calculations at the frequencies $\hbar \omega=0.10,0.30,0.50 \mathrm{MeV}$ have been presented.

One can see that all the potential energy surfaces are symmetrical with the $\varphi=0^{\circ}$ line. This means that the two chiral configurations with $\pm|\varphi|$ for a given tilted angle $\theta$ are identical. With the increasing frequency, the minima in the potential energy surfaces change from $\varphi=0^{\circ}$ to $\varphi \neq 0^{\circ}$. As discussed in Ref. [1], this implies the rotating mode changes from planar to aplanar rotation.

By minimizing the total Routhian $E^{\prime}(\theta, \varphi)$ with $\theta$ for given $\varphi$, the potential energy $V(\varphi)$ in the collective Hamiltonian (13) is obtained and shown in Fig. 2. Again, the potential energy is symmetrical about $\varphi=0^{\circ}$ in correspondence with the results shown in Fig. 1. For the frequency $\hbar \omega \leq 0.15 \mathrm{MeV}$, the potential $V(\varphi)$ is a harmonic oscillator type which has only one minimum at $\varphi=0^{\circ}$. This corresponds to the planar rotation [1]. For the frequency $\hbar \omega \geq 0.20 \mathrm{MeV}$, the potential $V(\varphi)$ has two symmetrical minima. This corresponds to the aplanar rotation [1]. Due to the appearance of the potential barrier which breaks the chiral symmetry, the stable chiral solutions are found in the body-fixed frame. The heights of barrier defined as $\Delta V=V(0)-V_{\min }\left(\right.$ in $\mathrm{MeV}$ ) with $V_{\min }$ being the value of the potential at the minimum presented also in the figure. It is found that the potential barrier increases with the cranking frequency, e.g., from $1 \mathrm{keV}$ at $\hbar \omega=0.20 \mathrm{MeV}$ to about $2 \mathrm{MeV}$ at $\hbar \omega=0.50 \mathrm{MeV}$. 

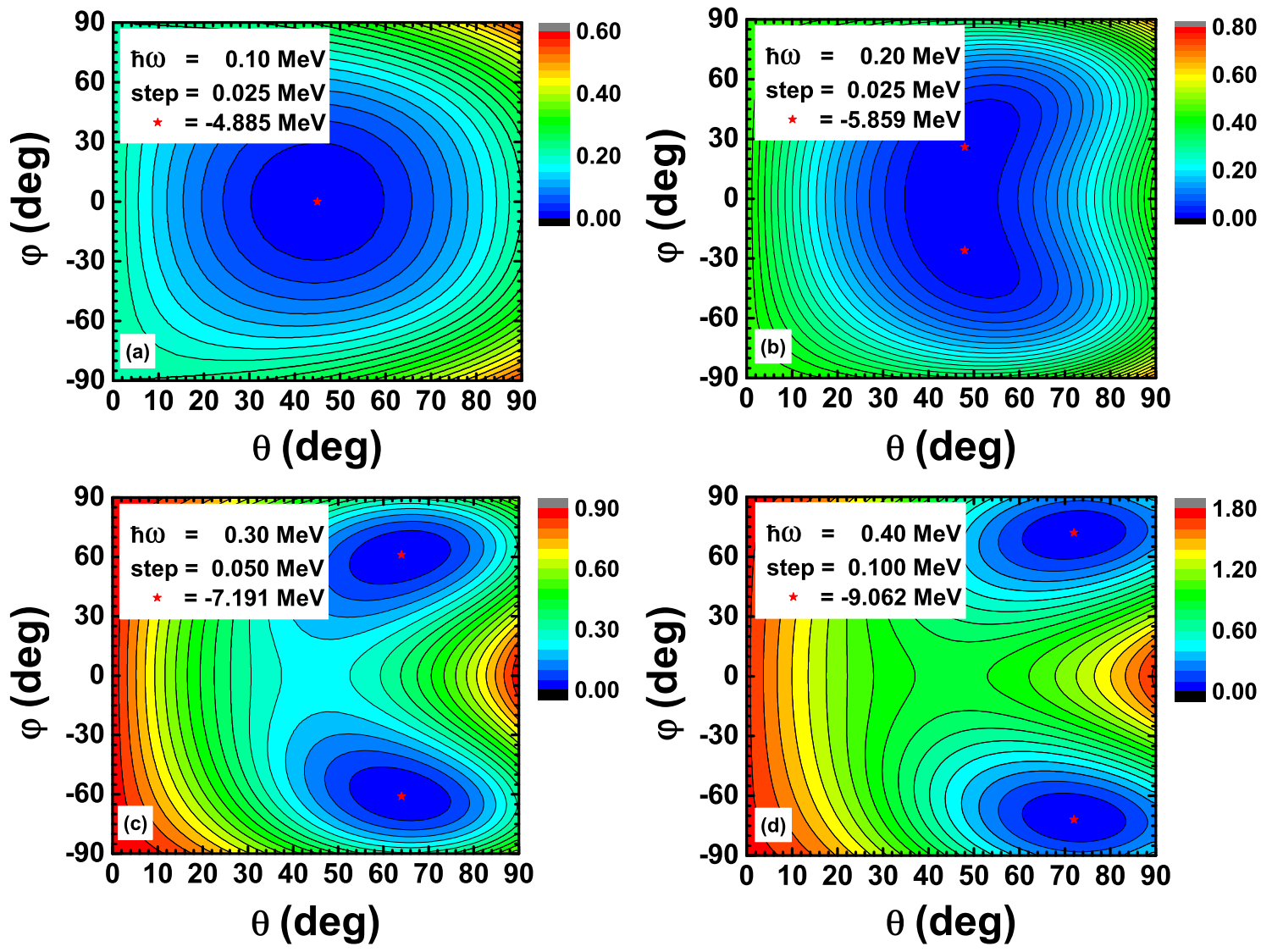

FIG. 1: (Color online) Total Routhian surface calculations for the $h_{11 / 2}$ proton particle and the $h_{11 / 2}$ neutron hole coupled to a triaxial rotor with $\gamma=-30^{\circ}$ at the frequencies $\hbar \omega=0.1,0.2,0.3$, 0.4 MeV. All energies are normalized with respect to the absolute minimum (star). The step is the energy difference between the contour lines.

\section{B. Mass parameter}

For chiral rotation with $\hbar \omega \geq 0.20 \mathrm{MeV}$, the chiral vibration frequency $\Omega$ in Eq. (22) is taken as $\Omega=0$, which results in the mass parameter

$$
B(\varphi)=2 \hbar^{2} \sum_{l \neq 0} \frac{\left|\frac{\partial \vec{\omega}}{\partial \varphi}\langle l|\hat{\vec{j}}| 0\rangle\right|^{2}}{\left(E_{l}-E_{0}\right)^{3}} .
$$

In Fig. 3, the mass parameter $B(\varphi)$ as a function of $\varphi$ for the chiral rotation cases is shown. It is seen that $B(\varphi)$ is symmetric with respect to $\varphi=0^{\circ}$ and increases dramatically when $\varphi$ approaches to $\pm 90^{\circ}$. In the interior part, $B(\varphi)$ is increased remarkably with $|\varphi|$ for $\hbar \omega \geq 0.35 \mathrm{MeV}$, while its dependence on $\varphi$ is weak for $\hbar \omega=0.25$ and $0.30 \mathrm{MeV}$. 


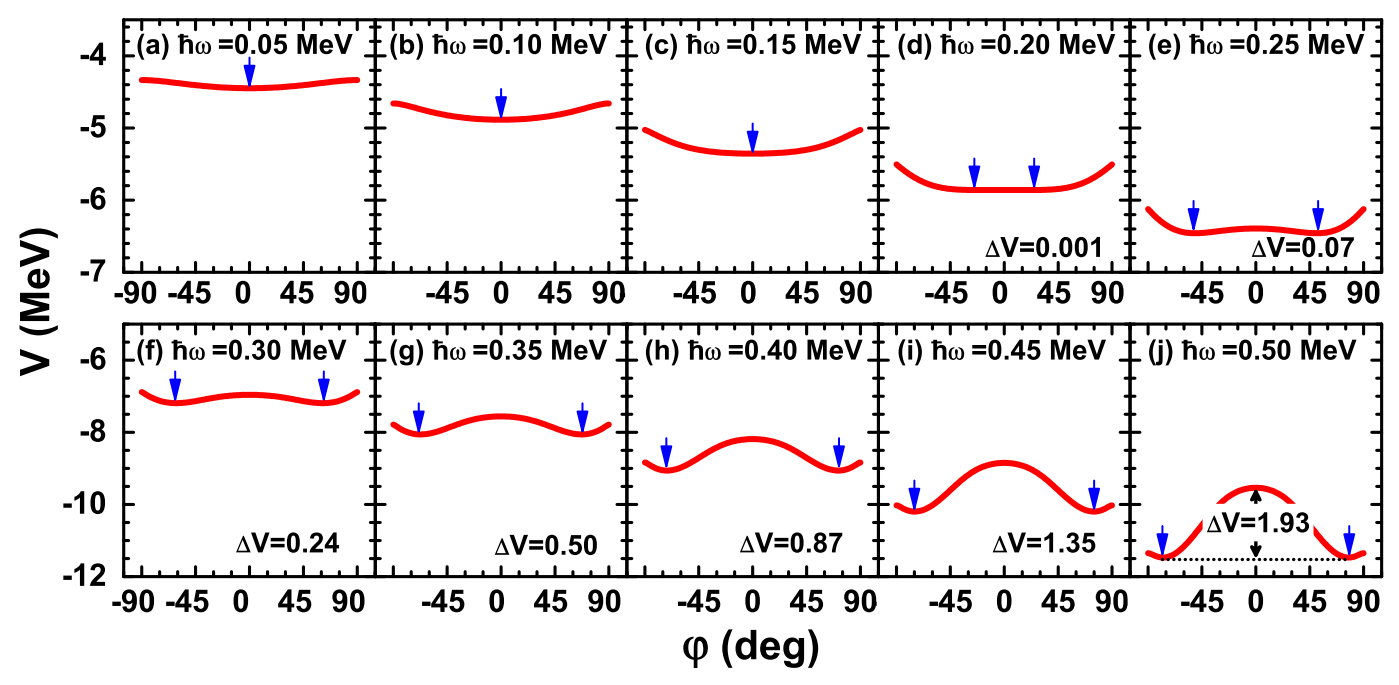

FIG. 2: (Color online) The potential energy $V(\varphi)$ as a function of $\varphi$ extracted from the total Routhian surface calculations. The arrow labels the position of the potential minimum $V_{\min }$. The potential barriers defined as $\Delta V=V(0)-V_{\min }$ (in $\mathrm{MeV}$ ) are also presented.

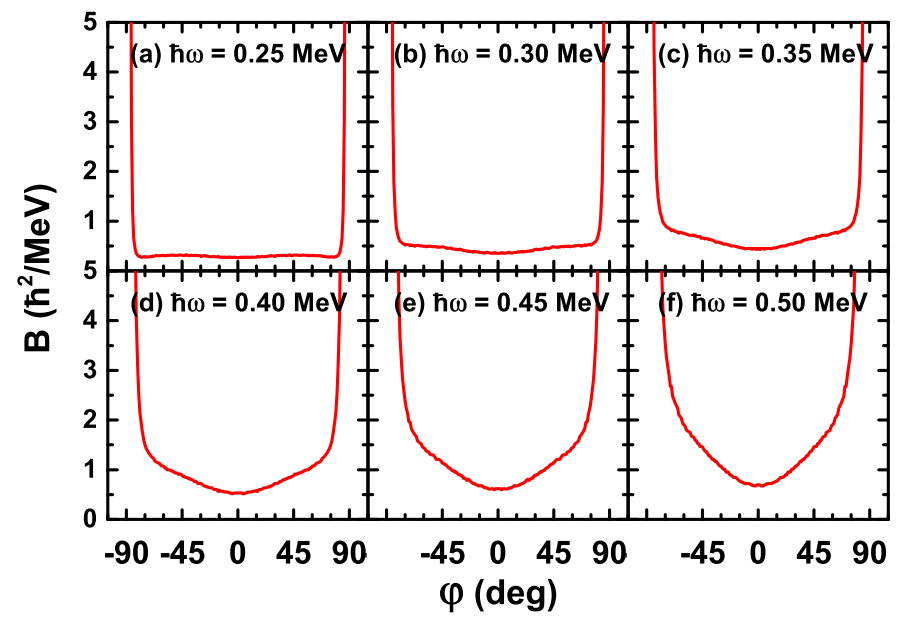

FIG. 3: (Color online) The mass parameter $B(\varphi)$ as a function of $\varphi$ for the chiral rotation cases obtained based on TAC.

\section{Energy levels}

The collective Hamiltonian can be constructed by Eq. (13) with the potential energy $V(\varphi)$ and mass parameter $B(\varphi)$ obtained. The diagonalization of the collective Hamiltonian yields the energy levels and wave functions for each cranking frequency. As $B(\varphi)$ is quite large at 
$\varphi \sim \pm 90^{\circ}$, a wall approximation is adopted. This corresponds to the choice of basis in Eq. (26).

In Fig. 4, the six lowest energy levels, labeled as 1-6, obtained from the collective Hamiltonian are shown together with the potential energy $V(\varphi)$. It is seen that with the increasing frequency, the three pairs of energy levels, i.e., levels 1 and 2, levels 3 and 4 as well as levels 5 and 6 , become close to each other. For example, the energy difference between levels 1 and 2 decreases from $1.19 \mathrm{MeV}$ at $\hbar \omega=0.25 \mathrm{MeV}$ to $0.01 \mathrm{MeV}$ at $\hbar \omega=0.50 \mathrm{MeV}$. Since the potential barrier becomes higher and wider with the increase of cranking frequency, the tunneling penetration probability is more and more suppressed. Therefore, the two levels tend to be more degenerate.
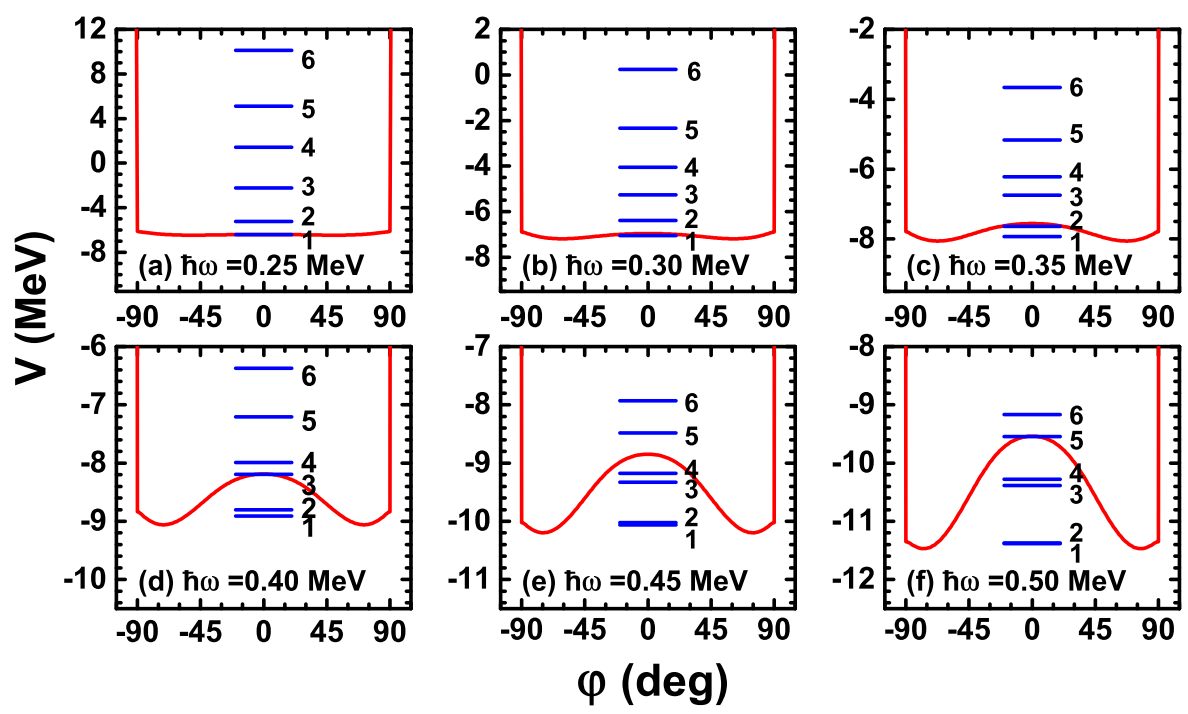

FIG. 4: (Color online) The six lowest energy levels, labeled as 1-6, obtained from the collective Hamiltonian. The potential energy $V(\varphi)$ is included as well.

This can be more clearly seen in Fig. 5 where the energy difference $\Delta E$ between the lowest two levels of the collective Hamiltonian is shown as a function of the potential barrier height $\Delta V$. It shows that with the increase of the cranking frequency, the potential barrier grows from $1 \mathrm{keV}$ to about $2 \mathrm{MeV}$, while the energy differences $\Delta E$ drops correspondingly from $1.19 \mathrm{MeV}$ to $0.01 \mathrm{MeV}$. 


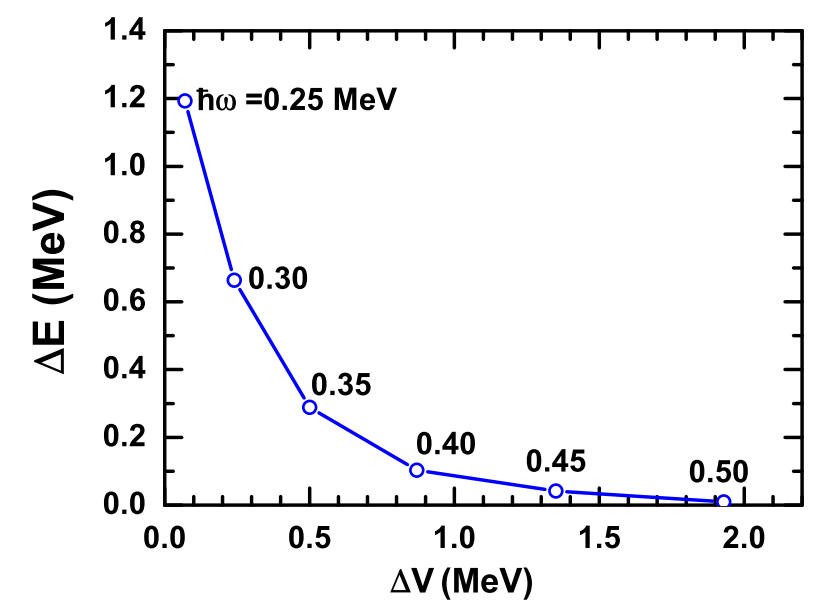

FIG. 5: (Color online) The energy difference $\Delta E$ between the lowest two levels 1 and 2 of the collective Hamiltonian as a function of the potential barrier height $\Delta V$.

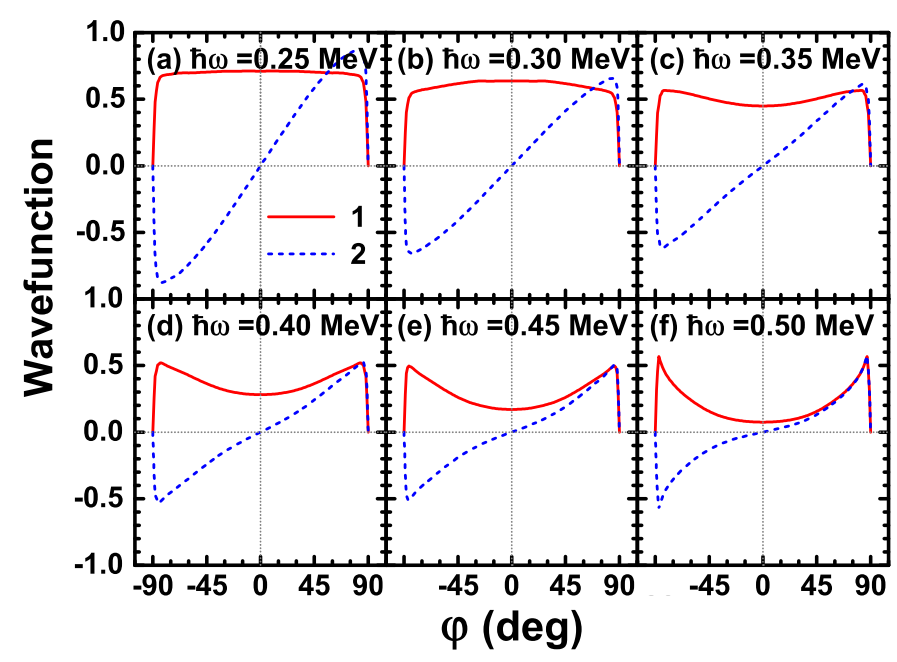

FIG. 6: (Color online) Wave functions $\psi(\varphi)$ for the lowest two levels 1 and 2 obtained from collective Hamiltonian.

\section{Wave function}

The wave functions $\psi(\varphi)$ for the lowest two levels are shown in Fig. 6 and the corresponding probability distributions determined as $|\psi(\varphi)|^{2}$ are shown in Fig. 7. It is found that the wave functions are symmetric for level 1 and antisymmetric for level 2 with respect to $\varphi \rightarrow-\varphi$ transformation. Thus the chiral symmetry broken in the aplanar TAC solutions is restored. It is also shown that the state with symmetric wave function is favored in energy 


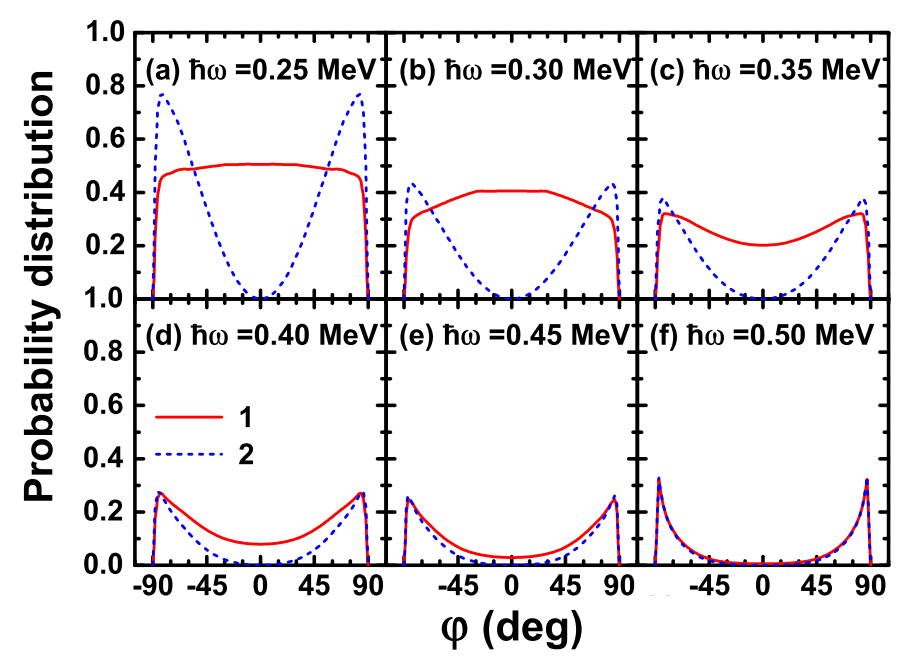

FIG. 7: (Color online) Probability distributions for the lowest two levels 1 and 2 calculated by $|\psi(\varphi)|^{2}$.

compared with the corresponding state with antisymmetric wave function.

For the frequency $\hbar \omega=0.25$ and $0.30 \mathrm{MeV}$, the wave function of the level 1 remains almost constant except at the border $\varphi= \pm 90^{\circ}$ which indicates that the fluctuation of the total angular momentum is large. The wave function of the level 2 shows peaks close to $\varphi= \pm 90^{\circ}$, which suggests that the chiral vibration plays an important role for the collective excitation at the beginning of the chiral rotation region. When the cranking frequency increases, the wave function of level 1 tends to show similar pattern. This implies the appearance of the nearly identical physical properties of these two levels and a good static chirality appears.

It is well known that the TAC results are given in the body-fixed frame and the two chiral partner solutions are identical. It cannot describe tunneling between the left- and rightoriented solutions. The chiral symmetry is thus broken and only one rotational band can be obtained. However, in the present formulation of the collective Hamiltonian, the results are given in the laboratory reference frame, which could describe the quantum tunneling between the two chiral solutions. Moreover, the chiral symmetry is restored in the present framework by considering the contributions from all the $\varphi$ directions in the potential energy surface. 


\section{E. Comparison with exact solutions}

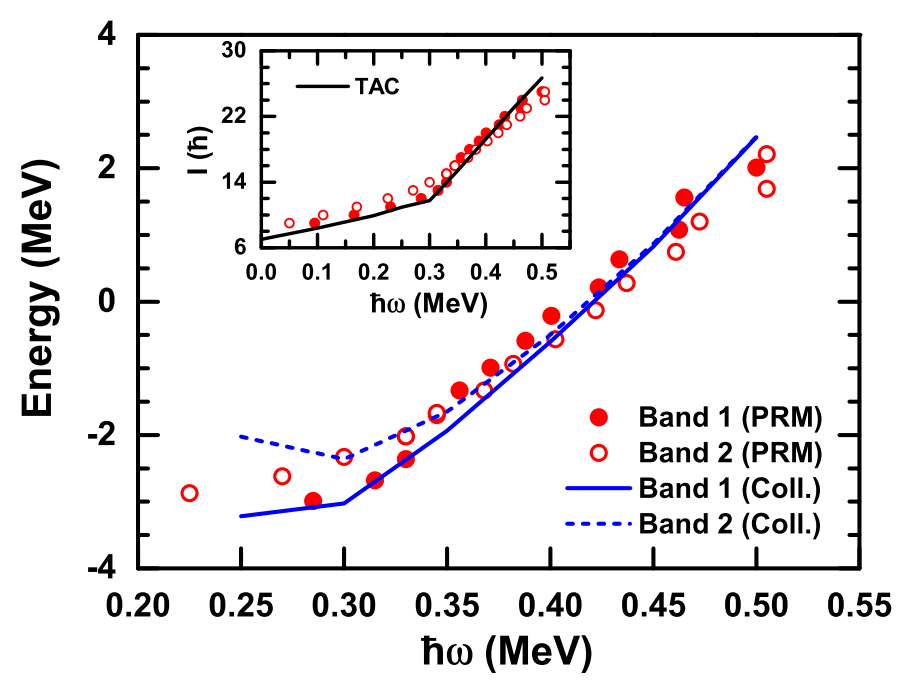

FIG. 8: (Color online) The energy spectra of the doublet bands obtained from the collective Hamiltonian in comparison with the exact solutions by the PRM. Inset: The spin $I(\omega)$ obtained from TAC in comparison with PRM. A similar $I(\omega)$ plot has been given in Ref. [1].

The exact solutions for the system discussed here can be obtained by the particle rotor model. In Ref. [1], the relation between the angular momentum $I$ and the rotational frequency $\hbar \omega$ as well as the intra band transition probabilities obtained from the TAC has been compared with the PRM results. It has been shown that the TAC solutions could reproduce the results of the yrast levels of the PRM calculation [1]. Here, in the inset of Fig. 8, the spin $I(\omega)$ obtained from TAC in comparison with PRM is shown once more, where the good agreement can be clearly seen.

In Fig. 8, the energy spectra of the doublet bands obtained from the collective Hamiltonian based on TAC are compared with the PRM results. One can see that apart from the agreement of collective Hamiltonian and PRM results for the yrast band, the partner band of PRM can also be reasonably reproduced by the collective Hamiltonian.

For the collective Hamiltonian results, the energy differences between the doublet bands become smaller with the increase of the cranking frequency. For the PRM results, however, the doublet bands become closer up to $\hbar \omega \sim 0.35 \mathrm{MeV}$ and the energy differences between the doublet bands continue to increase for the higher cranking frequency. As demonstrated 
both in PRM [4, 15, 16] and TAC+RPA [10] investigations, the doublet bands will attain a second chiral vibration character, which is not taken into account by the present collective Hamiltonian investigation.

The present comparison for the energy spectra is made with respect to rotational frequency $\hbar \omega$, rather than the observable of spin $I$. In the TAC approach, the angular momentum is not a good quantum number, and the diagonalization of the collective Hamiltonian is carried out for a given rotational frequency. Therefore, it is appropriate to compare the energy spectra with respect to the rotational frequency with the results of PRM. It can be seen from the inset of Fig. 8 that, for a certain spin, the rotational frequencies of the doublet bands 1 and 2 calculated by PRM may be quite different (for example, the differences are respectively $0.06,0.0,0.02$, and $0.04 \mathrm{MeV}$ at $I=12,16,20,24 \hbar)$. Here the fluctuation of the potential energy with $\theta$ is neglected and only the $\varphi$ is treated as a dynamical variable describing the transition from the left-handed to the right-handed system. The success of the collective Hamiltonian here guarantees its application for realistic TAC calculations.

\section{SUMMARY}

In summary, a collective model which is able to describe the chiral rotation and vibration is proposed and applied to a system of one $h_{11 / 2}$ proton particle and one $h_{11 / 2}$ neutron hole coupled to a triaxial rigid rotor. In this framework, it goes beyond the mean-field approximation, includes the quantum fluctuation in the chiral degree of freedom, and restores the

chiral symmetry. Based on the tilted axis cranking approach, both the potential energy and mass parameter as functions of $\varphi$ are obtained and included in the collective Hamiltonian. Diagonalizing the collective Hamiltonian with a box boundary condition, the energies and the wave functions of the collective states corresponding to the motion along the chiral degree of freedom are obtained. It is found that for chiral rotation, the partner states become more degenerate with the increase of the cranking frequency, and for the chiral vibrations, their important roles for the collective excitation are revealed at the beginning of the chiral rotation region. 


\section{ACKNOWLEDGMENTS}

This work was partly supported by the Major State 973 Program under Grant No. 2013CB834400, the National Natural Science Foundation of China under Grants No. 10975007, 10975008, 11105005, and No. 11175002, the Research Fund for the Doctoral Program of Higher Education under Grant No. 20110001110087, and the China Postdoctoral Science Foundation under Grant No. 2012M520101.

[1] S. Frauendorf and J. Meng, Nucl. Phys. A 617, 131 (1997).

[2] K. Starosta et al., Phys. Rev. Lett 86, 971 (2001).

[3] S. Mukhopadhyay et al., Phys. Rev. Lett. 99, 172501 (2007).

[4] B. Qi, S. Q. Zhang, J. Meng, S. Y. Wang, and S. Frauendorf, Phys. Lett. B 675, 175 (2009).

[5] V. I. Dimitrov, S. Frauendorf, and F. Dönau, Phys. Rev. Lett. 84, 5732 (2000).

[6] P. Olbratowski, J. Dobaczewski, J. Dudek, and W. Plociennik, Phys. Rev. Lett. 93, 052501 (2004).

[7] P. Olbratowski, J. Dobaczewski, and J. Dudek, Phys. Rev. C 73, 054308 (2006).

[8] J. Peng, J. Meng, and S. Q. Zhang, Phys. Rev. C 68, 044324 (2003).

[9] S. Q. Zhang, B. Qi, S. Y. Wang, and J. Meng, Phys. Rev. C 75, 044307 (2007).

[10] D. Almehed, F. Dönau, and S. Frauendorf, Phys. Rev. C 83, 054308 (2011).

[11] S. Frauendorf, Nucl. Phys. A 557, 259c (1993).

[12] K. Starosta, T. Koike, C. J. Chiara, D. B. Fossan, and D. R. LaFosse, Nucl. Phys. A 682, $375 c(2001)$.

[13] W. Pauli, in Handbuch der Physik, vol. XXIV, p. 120 (Springer Verlag, Berlin, 1933).

[14] D. J. Rowe, Nuclear Collective Motion: Models and Theory (Methuen, London, 1970).

[15] B. Qi, S. Q. Zhang, S. Y. Wang, J. M. Yao, and J. Meng, Phys. Rev. C 79, 041302(R) (2009).

[16] B. Qi, S. Q. Zhang, S. Y. Wang, J. Meng, and T. Koike, Phys. Rev. C 83, 034303 (2011). 